\title{
STEM CELL THERAPY IN THE TREATMENT OF BILATERAL ELBOW JOINT OSTEOARTHRITIS IN DOG
}

\author{
Uǵis Skangals, Agris Ilgažs \\ Latvia University of Life Sciences and Technologies, Latvia \\ ugis.skangals@gmail.com
}

\begin{abstract}
The aim of this pilot study was to assess whether a single mesenchymal stem cell intra-articular injection in the osteoarthritic joint gives a therapeutic effect. This trial study was made in order to better manage and structure further study on more dogs. Single injections of 3 million mesenchymal stem cell intra-articular injections were made bilaterally in the osteoarthritic elbow joints. The dog was examined before and after monthly stem cell therapy using stance analyser scales to determine the static weight of each limb. Weight balance between forelegs improved, but not significantly ( $>0.05)$. A radiographic examination was made for both elbow joints before stem cell therapy and the second and fifth month thereafter. Calculations of sub-trochlear sclerosis in percentage were made using mediolateral projections. Sub-trochlear sclerosis in percentage significantly decreased $(p<0.05)$ after stem cell therapy in both legs. Key words: stem cells, osteoarthritis, canine, elbow joint.
\end{abstract}

\section{Introduction}

Osteoarthritis (OA) is one of the most commonly diagnosed joint diseases in both humans and dogs. The prevalence of osteoarthritis in dogs is reported in the literature with conflicting values. Estimates have ranged from $6.6 \%$ based on primary-care data, to $20 \%$ based on referral data in the United Kingdom dog population. Assessment from North America report age specific prevalence values ranging from $20 \%$ in dogs older than 1 year up to $80 \%$ in dogs older than 8 years, based on radiological and clinical examination data from referral settings (O’Neill et al., 2018). Among dogs over one year of age, almost one out of every five has some degree of OA. Additionally, the morbidity in OA increases with age, and about $95 \%$ of OA cases occur in dogs over five years with dogs older than 10 years accounting for $50 \%$ of canine OA cases (Luo et al., 2018). OA is a common clinical disease in dogs affecting several tissues, including joint cartilage, subchondral bone, synovial membrane, and tendons (Luo et al., 2018). This musculoskeletal disease is related to chronic pain, lameness, loss of joint function and mobility, functional disability and reduced quality of life (Comblain et al., 2017). Pain results in both local and distant deterioration of the musculoskeletal system as a result of decreased and altered mobility (Cachon et al., 2018). OA induces joint swelling, pain, deformation, and effusions (Luo et al., 2018). The joint capsule also experiences a fibrotic redevelopment process, which becomes clinically apparent through the restricted mobility of the joint (Neumann \& Lauenstein-Bosse, 2018). OA is characterized by a gradual loss of cartilage and results in the development of osteophytes at the margins of the joints. The articular cartilage is damaged by a complex interaction of genetic, metabolic, biochemical and biomechanical factors, followed by activation of inflammatory responses involving the synovium, subchondral bone and cartilage. Pro- inflammatory cytokines and chemokines are plentiful in OA (Di Marzo et al., 2017).

Canine elbow dysplasia (CED) is the most common developmental disease of the elbow joint and results in forelimb lameness in medium and large breed, juvenile and adult dogs. CED is a syndrome, which consists of several conditions, including elbow joint incongruity (INC), ununited anconeal process (UAP), fragmentation of the medial coronoid process (FCP) of the ulna also named as coronoid dysplasia and osteochondritis dissecans (OCD) of the medial part of the humeral condyle. These conditions can occur as single traits or in combination and each can cause irreversible elbow OA (Theyse, 2018). Radiographic hallmarks of cartilage pathology include mainly joint space narrowing, subchondral sclerosis, subchondral cysts, osteophyte formation, and chronic inflammation of ligaments (Luo et al., 2018). The radiological findings of the elbow joints are according to the severity (size) of the presence of osteophytes and/or the presence of abnormalities reflecting the primary lesions UAP, OCD, FCP or Medial Coronoid Disease (MCD) and/or INC according to the International Elbow Working Group (IEWG) update in 2010. Grading elbow dysplasia according to the IEWG: Grade 0 - Normal elbow joint, no evidence of INC, sclerosis or arthrosis. Grade 1 - mild arthrosis; sclerosis of the base of the coronoid processes but trabecular pattern still visible, presence of osteophytes $<2 \mathrm{~mm}$. Grade 2 - moderate arthrosis and suspect primary lesion; step between radius and ulna $3-5 \mathrm{~mm}$ (INC), indirect signs for other primary lesion (UAP, FCP/MCD, OCD), presence of osteophytes $2-5 \mathrm{~mm}$, obvious sclerosis (no trabecular pattern) of the base of the coronoid processes. Grade 3 - severe arthrosis and evident primary lesion; presence of osteophytes $>$ $5 \mathrm{~mm}$; step between radius and ulna $>5 \mathrm{~mm}$ (obvious INC), obvious presence of a primary lesion (UAP, FCP/CD, OCD) (Hazewinkel, 2018). 
Articular cartilage is a highly specialised tissue with shock absorber function and enabling synovial joints to articulate with low frictional forces. Due to its avascular, aneural and alymphatic state, it has a limited repair potential (Goldberg et al., 2017).

Because of ineffective healing of chondral defects, there is a need to develop therapies to restore articular cartilage to near normal (Counsel et al., 2015). The best treatment of OA is focused on blocking the catabolic activity of cartilage and enhancing regeneration of normal cartilage. At the moment, therapies for the OA is focused on the reduction of pain and discomfort, prevention of further degeneration and improvement on functional movement. The most common treatment of OA is symptomatic and involves use of nonsteroidal anti-inflammatory drugs (NSAIDs), analgesics, and chondro-protectants, but it provides no diseasemodifying effect (Yun, Ku, \& Kwon, 2016). There is a growing interest in regenerative medicine: cell therapy, where cells are injected directly into the blood or into tissues. Stem cells are cells that have the ability to divide and transform into many different cell types in the body. Stem cells can be categorised as pluripotent and multipotent were pluripotent stem cells are harvested from embryonic sources and can develop into any type of cell in the body. Multipotent stem cells are taken from adults and can divide and develop into a more limited range of cell types. When stem cells divide, the new cells can either stay as stem cells or they can cultivate into a new type of cell with a more specific function (Goldberg et al., 2017). In early stages of life, each of these organs has a population of stem cells, called adult stem cells, that have roles in replenishing damaged cells by self-renewal and a unique ability to form the particular cell type it resides in. With age, the supply of these self-renewing stem cells significantly decreases by tenfold from new born to teenage years and again by teenage to old age (Hansen et al., 2018). For instance, adult stem cells isolated from various sources, mainly bone marrow (BM) and adipose tissue (AT) derived stem cells, have been widely used for the treatment of different animal diseases. They have the potential to differentiate into numerous cell types such as osteoblasts, adipocytes, cardiomyocytes, chondrocytes, hepatocytes, and brain cells (Sultana et al., 2018). The use of AT derived stem cells has gained popularity as alternative to BM derived stem cells (Balolong et al., 2016). Mesenchymal stem cell (MSC) is present in all tissues and organs. Their primary function is the replacement of dead cells in the physiological cell renewal processes. Besides, they may also replace dead cells in pathological situations, such as inflammation, ischemia or trauma. The MSC have the natural ability for multipotency, being capable of generating new cells of tissues derived from this germ layer. These cells, by action of hormones and growth factors, acquire morphophysiological aspects pertinent to their location within the body. According to the signalling factors that the MSC are exposed, different decisions may be taken, where the main ones are those that are involved in proliferation and cellular differentiation. The MSC capture and send molecular signals, which change the niche, either by modulating the immune system as providing mechanisms for tissue repair effectors, involving since activation of cell homing, cell apoptosis, induction of the formation of new blood vessels, and the healing process (Markoski, 2016). Other important features of MSCs are their anti-inflammatory and immuno-suppressive abilities enabling them to significantly attenuate the immune responses in the host. These cells along with soluble bioactive compounds are then able to inhibit the activation of $\mathrm{T}$ lymphocytes, B lymphocytes, and natural killer cells. Because of this important property, MSCs can be used in allogeneic treatment where the cells from one donor dog can be used to treat other dogs. In this regard, adult stem cell therapy, in the form of MSCs, has recently provided a new paradigm for treating chronic arthritic dogs from symptom management to stimulating regeneration of bones and cartilage, resulting in considerable improvements in quality of life. MSCs were able to aggregate, multiply, and bridge injured tissue by forming vasculaturecontrolled chondrocytes for cartilage formation and osteoblast for bone formation (Hansen et al., 2018). Intra-articular injection holds several potential advantages, including reduced recovery time and cost (Counsel et al., 2015). In veterinary medicine, the number of animals already treated provides a substantial basis for assessing the effectiveness of cell therapy in the treatment of a large number of diseases. However, in general, the therapeutic issues involving the use of stem cells to regenerate tissue still have not been fully understood (Markoski, 2016).

The aim of this pilot study was to assess whether a single mesenchymal stem cell intra articular injection in the osteoarthritic joint gives a therapeutic effect. And if it does, then in the near future conduct a more complex study, with more examinations and observations on more dogs to better evaluate therapeutic effect of this type of treatment.

\section{Materials and Methods}

The research was carried out at a small animal veterinary clinic of the Latvia University of Life Sciences and Technologies from April to September, 2018. The research was done on one 4 year old dog with bilateral elbow joint dysplasia (ED) and OA. The dog was constantly lame and stiff on both forelegs. The symptoms were more pronounced on the left foreleg. According to the dog owner, lameness and stiffness was observed from the age of 3 months. At the age of 
5 months a radiographic examination was made and ED diagnosed with primary lesions UAP and INC, and signs of OA - osteophytes and subtrochlear sclerosis. At the age of 6 months, an athrotomy was made, anconeal process removed and dynamic, proximal ulna osteotomy was performed to the right elbow joint. The owner of the animal voluntarily agreed to participate in the study.

The research plan was to make examinations before stem cell therapy and repeat examinations once a month for five months. For the first month, the dog was examined on a 'ReHab KRUUSE E1061' stance analyser. The stance analyzer measures the distribution of body weight on each limb when standing and may allow for subtle differences to be seen between the outcomes of various surgical procedures and allow assessment of progression of the disease or recovery over time (Wilson, 2018). Five measurements were recorded. For sedation, Butorphanol $0.4 \mathrm{mg} \mathrm{kg}^{-1}$ intravenous (i.v.) injections were made as a pre-medication and slow propofol i.v. injection made until the palpebral reflex disappeared. Radiographic examination for both elbow joints was made. A mediolateral projection was made positioning the dog in lateral recumbency as well as a craniocaudal projection made positioning the dog in ventral recumbency. After radiographic examination, the injection site preparation and stem cell intraarticular injection was made in both elbow joints. The 'CaniCell' stem-cell product from the company 'MEDREGO' containing 3 million cells was used. The lateral site of the elbow was clipped (clipper blade $40 \#)$. Preparation of the injection site was made by using alcohol and chlorhexidine swabs. The tube with the stem cells was manually defrosted and transferred to a sterile syringe. An intra-articular injection of aspirated joint fluid $(\sim 1.0 \mathrm{~mL})$ was then made to the elbow joint and the syringe was removed from the needle (the needle was kept in the joint). The syringe with the stem cell product was added to the needle and cells injected into the joint. The syringe with needle was removed from the joint and a slight massage on the injection site was made. The same procedure was repeated for the other elbow.

For the second, fourth and fifth visit, the dog was examined on the stance analyser. Each time five measurements were recorded. For the third and sixth visit, the dog was examined on the stance analyser and radiographic examination for mediolateral and ventrodorsal projections for both elbow joints were made. The dog was obedient and calm, so radiographic examination was made without sedation.

Statistical analysis

For every stance analyser examination, five measurement records were made. By using descriptive statistics, average values for each leg were determined from five measurements taken each time. These were used to calculate the weight difference between left and right foreleg as well as the weight difference between both forelegs and hind-legs. Graphs were made from the data obtained. Covariance analysis using a 95\% significance level was used to statistically estimate the monthly data.

To evaluate radiographic examinations, measurements of subtrochlear sclerosis (STS) were made using measurement of STS as a $\%$ of the distance between 2 standardized radiographic landmarks (\% STS) (Smith et al., 2009). Each flexed mediolateral projection was assessed for presence of STS. When present, STS was quantified as a percentage of the length between 2 fixed points. To measure \% STS, a line was created perpendicular to the most caudal margin of the ulnar proximal metaphyseal cortex and to the most proximo-caudal aspect of the radial head (Y). The STS caudal border which constituted a subjective radiographic assessment of the junction between sclerotic and normal trabecular bone pattern, was created along line to most proximo-caudal aspect of the radial head $(\mathrm{X})$. The distance $\mathrm{X}$ was expressed as a percentage of the total distance $\mathrm{Y}$. The $\%$ STS was calculated as 100(X/Y) (Fitzpatrick et al., 2009).

The data obtained was displayed in graphs. Covariance analysis using a 95\% significance level was used to statistically estimate the data over the months.

\section{Results and Discussion}

Comparison of monthly weight shows that the difference between forelegs decreases. There is no significant change in the statistical analysis of the data $(p>0.05)$. Representing the data in the graph (Figure 1 ), there is a downward linear trend. Weight symmetry between forelegs equalize, which shows a slight (though not significant) improvement.

Weight difference in percentage between forelegs and hind legs before stem cell injection was $12 \%$, after one month $19 \%$, after two months $23 \%$, after three months $10 \%$, after fore month $11 \%$ and five months after stem cell therapy it was $20 \%$. Comparing monthly weight difference between forelegs and hind legs there is no significant change in the statistical analysis of the data $(\mathrm{p}>0.05)$.

Stance analyser is a simple and good way to evaluate distribution of body weight on each limb at a stand; thus it can be determined on which limb it is more painful to lean on, and in movement, it will show like lameness. With this examination it can be evaluated if pain reduces in time after therapy. Results of this study shows a slight improvement in forelimb weight symmetry and it means that the legs are less painful. Previous studies show that the stem cell treatment resulted in pain and functional improvement. Based 


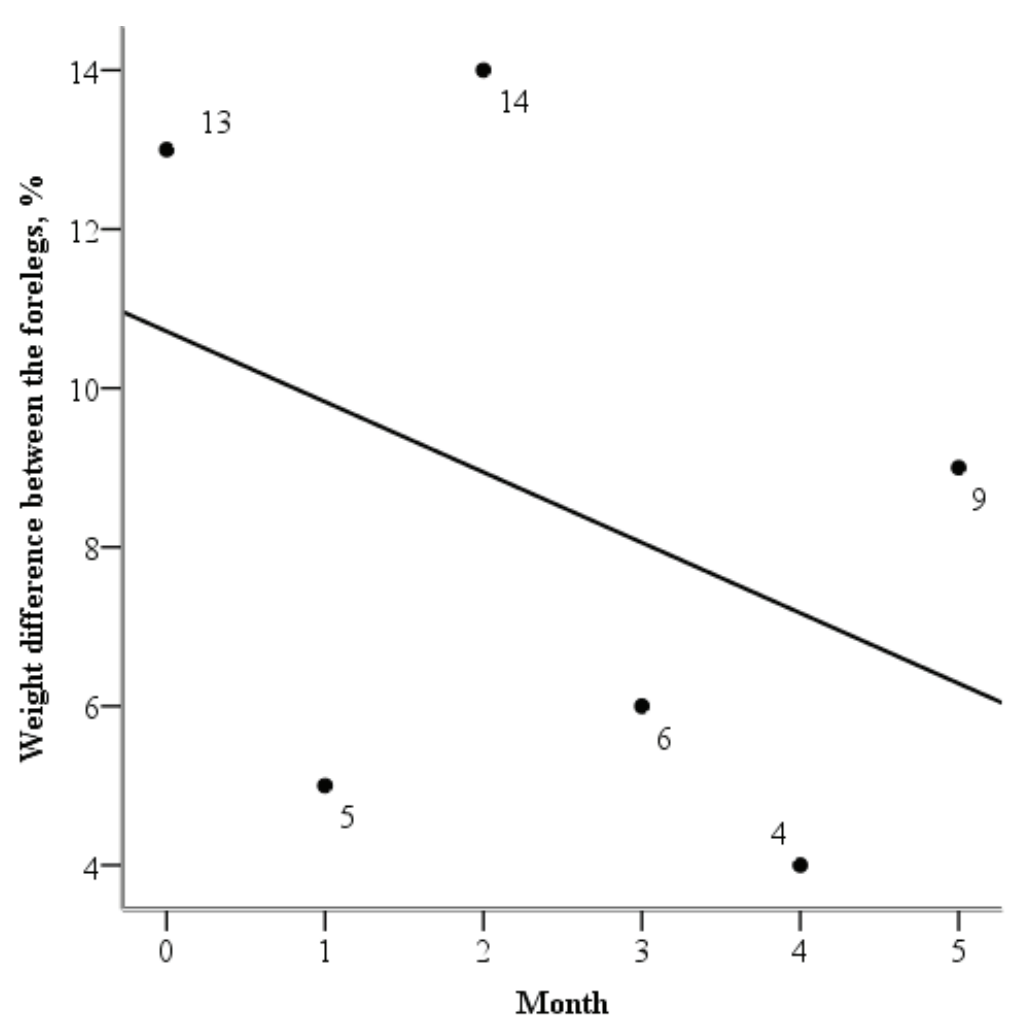

Figure 1. Weight difference between the right and left forelegs in months.

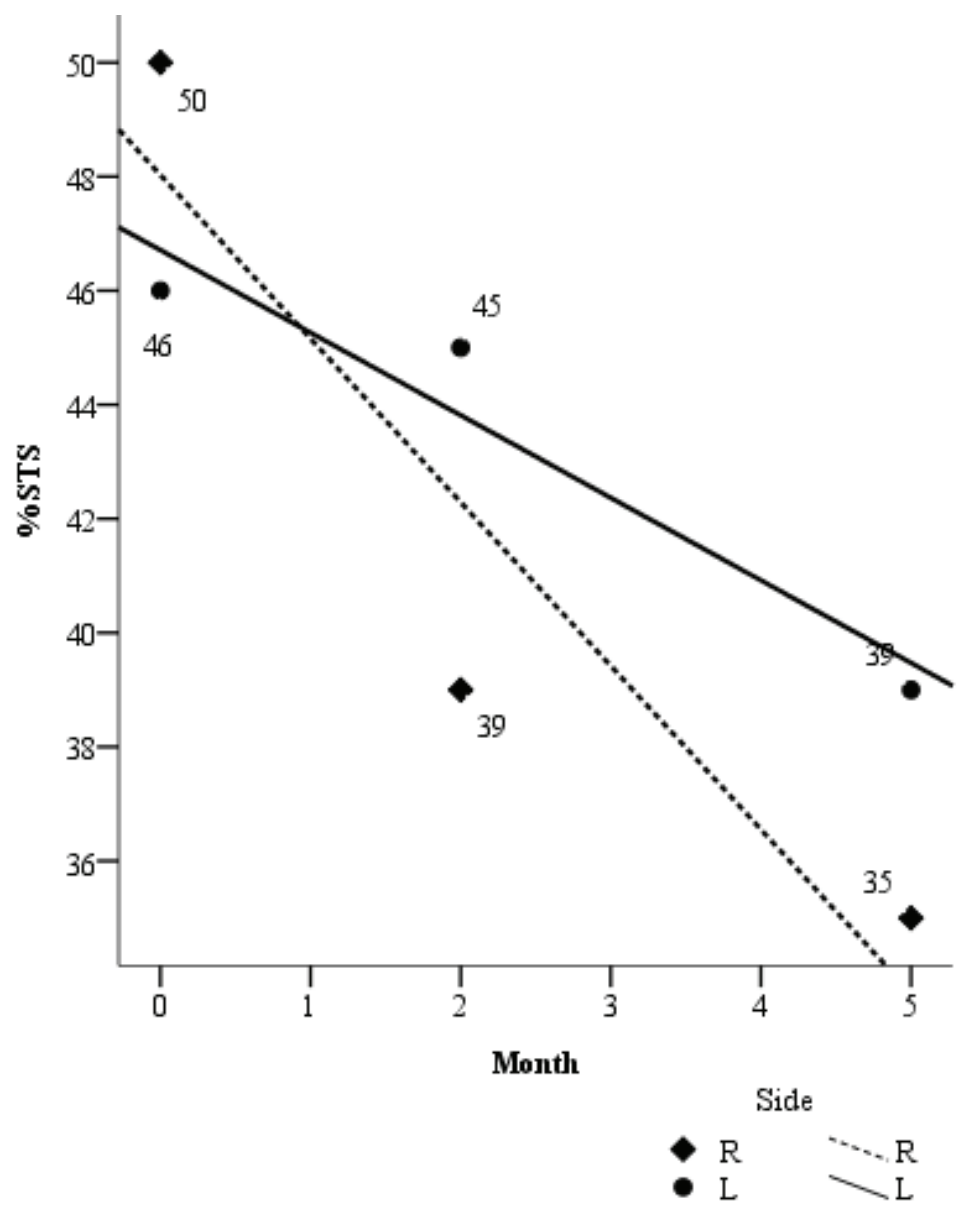

Figure 2. Right (R) and left (L) elbow \%STS in months. 
on the owner evaluations, the stem cell intraarticular injection had a significant effect on the dog's lameness during walking without any further medication for at least a one-year period (Huguenin et al., 2016; Kriston-Pál et al., 2017). Because the dog had bilateral elbow joint ostesteoarthritis and treatment was made on both elbow joints, results on the stance analyser are not seen well. In the basic study, dogs with unilateral elbow osteoarthritis should be definitely selected.

Evaluating \% STS changes in the right elbow joint in months acquires that STS reduced from $50 \%$ to $35 \%$ and in the left elbow joint it reduced from $46 \%$ to $39 \%$ (Figure 2). The results of the statistical analysis show a significant change in \% STS compared in months $(\mathrm{p}=0.044)$.

First radiological findings of elbow OA is STS and osteophytes. The stages of severity of elbow dysplasia developed by IEWG are determined by radiographic assessment of the presence of STS, the size of the osteophytes height, INC distance and the presence of primary pathology (UAP, FCP / MCD, OCD). In this study measurements of $\%$ STS were made. After stem cell therapy \% STS significantly $(p<0.05)$ decreased in both elbows. Reduction of STS is one of disease modifying effects. Previous studies show similar acknowledgement, stem cell treatment gives a disease modifying effect that stimulates regeneration of bones and cartilage, resulting in considerable improvements in life quality (Hansen et al., 2018; Huguenin et al., 2016). To evaluate \% STS importance better, in the basic study, it is necessary to observe it in a control group in elbow joints without osteoarthritis and without treatment. It is mentioned in other research, clinical importance of \% STS remains unknown (Smith et al., 2009).

\section{Conclusions}

1. Comparison of monthly distribution of body weight on each limb using stance analyser, weight symmetry between forelegs equalize, which shows a slight, but not significant, changes.

2. Comparing monthly weight difference between forelegs and hind legs there are no significant changes.

3. In radiographs evaluating $\% \mathrm{STS}$ changes in elbow joints in months acquires that \%STS significantly decreased. This may indicate that inflammation in the joint reduces, but at the moment there is no information that $\%$ STS correlates with severity of $\mathrm{OA}$ and stage of the disease.

4. Taking into account the results obtained in the pilot study, it is worth to perform the basic study on more dogs including more examinations, assessments, and animal owner questionnaires to better and more thoroughly evaluate changes after stem cell therapy.

\section{References}

1. Balolong, E., Lee, S., Nemeno, J.G., \& Lee, J.I. (2016). Are they really stem cells? Scrutinizing the identity of cells and the quality of reporting in the use of adipose tissue-derived stem cells. Stem Cells International, 2016. DOI: $10.1155 / 2016 / 2302430$.

2. Cachon, T., Frykman, O., Innes, J.F., Lascelles, B.D.X., Okumura, M., Sousa, P., ... Van Ryssen, B. (2018). Face validity of a proposed tool for staging canine osteoarthritis: Canine OsteoArthritis Staging Tool (COAST). Veterinary Journal, 235, 1-8. DOI: 10.1016/j.tvj1.2018.02.017.

3. Comblain, F., Barthélémy, N., Lefèbvre, M., Schwartz, C., Lesponne, I., Serisier, S., ... Henrotin, Y. (2017). A randomized, double-blind, prospective, placebo-controlled study of the efficacy of a diet supplemented with curcuminoids extract, hydrolyzed collagen and green tea extract in owner's dogs with osteoarthritis. BMC Veterinary Research, 13(1), 1-11. DOI: 10.1186/s12917-017-1317-8.

4. Counsel, P.D., Bates, D., Boyd, R., \& Connell, D.A. (2015). Cell Therapy in Joint Disorders. Sports Health, 7(1), 27-37. DOI: 10.1177/1941738114523387.

5. Di Marzo, V., Valastro, C., Di Bello, A., Campanile, D., Marinaro, M., Verde, R., ... Piscitelli, F. (2017). Characterization of endocannabinoids and related acylethanolamides in the synovial fluid of dogs with osteoarthritis: a pilot study. BMC Veterinary Research, 13(1), 1-5. DOI: 10.1186/s12917-017-1245-7.

6. Fitzpatrick, N., Smith, T.J., Evans, R.B., \& Yeadon, R. (2009). Radiographic and arthroscopic findings in the elbow joints of 263 dogs with medial coronoid disease. Veterinary Surgery, 38(2), 213-223. DOI: 10.1111/j.1532-950X.2008.00489.x.

7. Goldberg, A., Mitchell, K., Soans, J., Kim, L., \& Zaidi, R. (2017). The use of mesenchymal stem cells for cartilage repair and regeneration: A systematic review. Journal of Orthopaedic Surgery and Research, 12(1), 1-30. DOI: 10.1186/s13018-017-0534-y.

8. Hansen, P., Shah, K., Malin, M., Ferguson, R., Boyd, R., Roic, I., ... Sumer, H. (2018). Outcome of Allogeneic Adult Stem Cell Therapy in Dogs Suffering from Osteoarthritis and Other Joint Defects. Stem Cells International, 2018, 1-7. DOI: 10.1155/2018/7309201.

9. Huguenin, L., Shah, K., Bates, D., Freitag, J., Barnard, A., Boyd, R., \& Tenen, A. (2016). Mesenchymal stem cell therapy in the treatment of osteoarthritis: reparative pathways, safety and efficacy - a review. BMC Musculoskeletal Disorders, 17(1), 1-13. DOI: 10.1186/s12891-016-1085-9. 
10. Theyse, L.F.H. (2018). Clinical, diagnostic and pathological findings in Canine Elbow Dysplasia. IEWG Proceedings 32. Retrieved March 9, 2019, from http://www.vet-iewg.org/wp-content/uploads/2018/09/ IEWGproceedings2018A2.pdf.

11. Hazewinkel, H.A.W. (2018). Screening for Elbow Dysplasia, grading according to the IEWG. IEWG Proceedings 33. Retrieved March 9, 2019, from http://www.vet-iewg.org/wp-content/uploads/2018/10/ IEWGproceedings2018Singapore.pdf.

12. Kriston-Pál, É., Czibula, Á., Gyuris, Z., Balka, G., Seregi, A., Sükösd, F., ... Monostori, É. (2017). Characterization and therapeutic application of canine adipose mesenchymal stem cells to treat elbow osteoarthritis. Canadian Journal of Veterinary Research $=$ Revue Canadienne de Recherche Veterinaire, 81(1), 73-78. Retrieved March 9, 2019, from http://www.ncbi.nlm.nih.gov/pubmed/28197017\%0Ahttp:// www.pubmedcentral.nih.gov/articlerender.fcgi?artid=PMC5220603.

13. Luo, D., Li, D., Zhan, X., Wang, B., Ji, H., Li, S., ... Zhang, B. (2018). Evaluation of the Curative Effect of Umbilical Cord Mesenchymal Stem Cell Therapy for Knee Arthritis in Dogs Using Imaging Technology. Stem Cells International, 2018, 1-12. DOI: 10.1155/2018/1983025.

14. Markoski, M.M. (2016). Advances in the Use of Stem Cells in Veterinary Medicine: From Basic Research to Clinical Practice. Scientifica, 2016, 1-12. DOI: 10.1155/2016/4516920.

15. Neumann, S., \& Lauenstein-Bosse, S. (2018). Evaluation of transforming growth factor beta 1 in dogs with osteoarthritis. Open Veterinary Journal, 8(4), 386. DOI: 10.4314/ovj.v8i4.6.

16. O’Neill, D.G., Brodbelt, D.C., Summers, J.F., Church, D.B., Collins, L.M., Anderson, K.L., ... Zulch, H. (2018). Prevalence, duration and risk factors for appendicular osteoarthritis in a UK dog population under primary veterinary care. Scientific Reports, 8(1), 1-12. DOI: 10.1038/s41598-018-23940-z.

17. Smith, T.J., Fitzpatrick, N., Evans, R.B., \& Pead, M.J. (2009). Measurement of ulnar subtrochlear sclerosis using a percentage scale in labrador retrievers with minimal radiographic signs of periarticular osteophytosis. Veterinary Surgery, 38(2), 199-208. DOI: 10.1111/j.1532-950X.2008.00488.x.

18. Sultana, T., Lee, S., Yoon, H.-Y., \& Lee, J.I. (2018). Current Status of Canine Umbilical Cord BloodDerived Mesenchymal Stem Cells in Veterinary Medicine. Stem Cells International, 2018, 1-14. DOI: $10.1155 / 2018 / 8329174$.

19. Wilson, M.L. (2018). Interday and intraday stance analysis variability in dogs with hindlimb lameness and comparison of the effect of dog, surgeon, and TPLO surgical procedure variables on improvement of eightweek post-operative static weight-bearing. 300.

20. Yun, S., Ku, S.K., \& Kwon, Y.S. (2016). Adipose-derived mesenchymal stem cells and platelet-rich plasma synergistically ameliorate the surgical-induced osteoarthritis in Beagle dogs. Journal of Orthopaedic Surgery and Research, 11(1), 1-12. DOI: 10.1186/s13018-016-0342-9. 